\title{
LA LEY 1620 DE 2013 Y LA POLÍTICA PÚBLICA EDUCATIVA DE CONVIVENCIA ESCOLAR EN COLOMBIA: ENTRE LA FORMALIDAD JURÍDICA Y LA REALIDAD SOCIAL *
}

\section{The Act 1620 of 2015 and the educational public policy of school coexistence in Colombia: between legal formality and social reality}

Henry Bocanegra Acosta **

Carlos Herrera Castillo***

Recepción: 21 de marzo de 2017 Aceptación: 12 de junio de 2017.

DOI: http://dx.doi.org/10.21017/Rev.Repub.2017.v23.a36

\section{RESUMEN}

Artículo producto de la labor investigativa sobre la política pública educativa de atención de las violencias que afectan a la escuela colombiana, niveles de preescolar, básica y media. Desde una conceptualización y contextualización del problema del acoso y la violencia escolar se avanzó en el reconocimiento y valoración de los lineamientos de la política pública respectiva y de sus principales desarrollos normativos legales y reglamentarios ocurridos.

* Artículo en colaboración, producto de la investigación desarrollada por los autores, quienes cooperaron en el análisis teórico y de la información empírica desde sus grupos, proyectos y experiencias investigativas en el referido campo de estudio. Colaboró con el cotejo de fuentes y aporte en el proceso de sistematización de la información el estudiante Joan Sebastián Marín Montenegro, integrante del semillero de investigación del Grupo de Investigaciones Socio Jurídicas (GISJ), proyecto Derechos, políticas púbicas y movimientos sociales, eje temático Derecho a la educación y violencia.

** Doctor en Sociología Jurídica e Instituciones Políticas, de la Universidad Externado de Colombia. Especialista y magíster en Administración Pública, Escuela Superior de Administración Pública (ESAP). Abogado de la Universidad Libre. Licenciado en Ciencias de la Educación, especialidad sociales. Docente investigador de la Universidad Libre, Grupo de Investigaciones Socio Jurídicas; docente de la Corporación Universitaria Republicana.

*** Doctorante en Derecho, Universidad Libre, Sede Principal. Magíster en Derecho, Universidad Nacional de Colombia. Especialista en Derecho Público, Universidad Externado de Colombia. Abogado, Universidad de Cartagena (Colombia). Docente del área de investigación de la Universidad Libre, Sede Principal, integrante del Grupo de Investigaciones Socio Jurídicas (GISJ) del Centro de Investigaciones Socio Jurídicas de la Universidad Libre, Sede Principal; reconocido y categorizado en A por Colciencias como Grupo Colombiano de Investigación Científica, Tecnológica e Innovación. 
El problema de investigación se sintetiza en una pregunta problematizadora que se plantea de la siguiente manera: ¿Qué elementos, instrumentos o mecanismos se introducen o innovan con la Ley 1620 de 2013 en materia de prevención y atención del acoso y de las diversas formas de violencia que afectan los ambientes escolares y los derechos humanos, sexuales y reproductivos de niños, niñas y adolescentes en el contexto socioeducativo colombiano? Metodológicamente es una investigación realizada desde un enfoque cualitativo, de carácter descriptivo y explicativo, realizada a partir de fuentes primarias y secundarias, que utilizó el método analítico, la hermenéutica jurídica y la síntesis. Desde allí se puede concluir que la Ley 1620 del 2013 creó un sistema no solamente para atender los hechos o situaciones de violencia escolar sino que básicamente lo define y estructura para desarrollar acciones de promoción de los derechos humanos sexuales y reproductivos, para prevenir las situaciones de violencia en la escuela y para hacer los seguimientos que cada caso requiera de acuerdo a su complejidad o gravedad.

Palabras clave: política educativa, convivencia escolar, acoso y violencia escolar, derecho a la educación.

\section{ABSTRACT}

This article is a product of the research on the educational public policy regarding violence management in Colombian pre-school, primary and secondary systems. The outset of this research reviews the concept and the context of violence and bullying within schools. This made headway regarding the acknowledge of public policy guidelines and its main legal developments. The research problem is aimed to answer the following question: Which elements, instruments or mechanisms have Act 1620 of 2016 introduced regarding the prevention and management problem of school coexistence and different ways of violence experienced within the country?

The methodology of this research was focused on a qualitative approach using primary and secondary sources and also an analytical method, the hermeneutics and the synthesis. This allowed concluding that Act 1620 of 2013 created a system to deal with the issues of school violence. Also, Law 1620 of 2013 outlined the situations of school violence and structure some actions of human sexual rights sensibilization to prevent violence at school and to follow up the cases according to their complexity and importance.

Key words: educational policy, school coexistence, school harassment and violence, the right to education. 


\section{INTRODUCCIÓN}

Este artículo es producto de una propuesta investigativa desde la cual se pretende indagar sobre el sentido, alcance, posibilidades y límites de la política pública educativa de atención de las violencias que afectan a las instituciones educativas en los niveles de básica y media en el contexto socio-educativo colombiano actual.

Es necesario reconocer las dinámicas sociales, culturales y pedagógicas generadoras de situaciones de conflicto y violencia en la escuela colombiana; conceptualizar sobre sus tipologías, formas, lógicas y efectos sobre la capacidad y posibilidades de la escuela, pero igualmente resulta pertinente abordar el análisis de los esquemas normativos y acciones que emprende el Estado, en sus diferentes niveles y entidades competentes, para atender la referida problemática y asegurar un clima y entorno escolar que favorezcan una educación de calidad y el respeto de los derechos sociales que le asisten a los diferentes estamentos de las comunidades educativas. Importante reconocer la envergadura del problema y desde allí valorar las acciones de las autoridades públicas.

El País Vasco ha alcanzado 15\% de presencia del matoneo en las escuelas, mientras que en Colombia se habla de $30 \%$ de jóvenes intimidados, cifra bastante similar a la de Estados Unidos (Manjarrés, 2012, p. 361).

Desde esta iniciativa académica se tuvo el propósito de valorar los fundamentos conceptuales y desarrollos jurídicos contemporáneos que se han dado en el marco de la política pública educativa de convivencia escolar en Colombia, especialmente lo que corresponde al complejo institucional construido desde la Ley 1620 de 2013, por la cual se crea el sistema nacional de convivencia escolar y formación para el ejercicio de los derechos humanos, la educación para la sexualidad y la prevención y mitigación de la violencia escolar. Una norma que ha generado abundante discusión, propuestas y contrapropuestas dado su alcance e incidencia sobre la organización institucional y pedagógica de las instituciones educativas. De otra parte, existen serias expectativas, dudas y temores entre los docentes y directivos docentes en la medida que les ha correspondido asumir la aplicación de los numerosos protocolos y procedimientos que dicha ley establece para atender las diversas tipologías de conflicto presentes cotidianamente en el ámbito escolar.

La expedición de la Ley 1620 de 2013 y del Decreto 1965 de 2013, reglamentario de esta ley, redefinen la arquitectura institucional del sector educativo en lo correspondiente a la atención de las diversas formas de violencia escolar. Independiente de las críticas que ha suscitado desde el mundo académico, dirigentes sindicales e integrantes de las propias instituciones educativas obje- 
to de su aplicación, esta norma representa un hito y un indiscutible referente teórico y práctico para la prevención y atención de este flagelo. De todos modos se tiene que reconocer que las normas por sí solas no resuelven los problemas sociales; el fetichismo jurídico no contribuye a nada. Las normas son simples referentes para adelantar acciones de mayor calado (políticas públicas), con las que se pretende atender necesidades o demandas sociales; con todo, resulta pertinente señalar que «el Estado no logra resolver, mediante la expedición de normas, todas las necesidades que se le plantean» (Molina Peláez, 2017, p. 66).

Colombia es un país que ha atravesado una prolongada trayectoria de conflagración endémica permanente que se extiende incluso hasta el período actual (Barragán Ronderos, 2016. p. 40). La violencia es una situación estructural y frecuente que ha venido afectando desde hace más de 50 años el devenir social, político, económico y cultural del pueblo colombiano. Como fenómeno estructural y habitual, la violencia ha permeado el ordenamiento institucional, la acción pública, las costumbres políticas, el actuar cotidiano de los ciudadanos, jóvenes y niños. La escuela colombiana ha soportado los avatares propios de una sociedad con altos niveles de violencia, inequidad, desigualdad y limitaciones institucionales y presupuestales. Resulta adecuado adelantar indagaciones que ausculten la realidad de los ambientes y contextos escolares, de las formas que asumen las violencias en las escuelas colombianas, pero especialmente que verifiquen sobre el sentido, alcance, posibilidades y efectos de la política pública y los desarrollos normativos correspondientes. Estamos de acuerdo con el planteamiento de Alexandra Marthe Manjarrés cuando expone:

Es posible cambiar o incidir para alterar o modificar los factores que contribuyen a producir respuestas violentas, ya sea los dependientes de la actitud y el comportamiento, o los relacionados con situaciones sociales, económicas, políticas y culturales más amplias. Los ejemplos de resultados exitosos en este sentido pueden encontrarse en todo el mundo, desde las acciones individuales y comunitarias en pequeña escala, hasta las iniciativas nacionales de política y legislativas (Manjarrés, 2012, p. 362).

La violencia se viene mostrando como una realidad que afecta el cumplimiento de la función social que le corresponde a la escuela colombiana y los derechos garantías y libertades de 10.203.000 estudiantes matriculados en diferentes instituciones colombianas en el 2016, de los cuales, según datos de la Ministra de Educación Nacional, Yaneth Giha, 7.600.000 están en instituciones educativas oficiales; 711.000 en matrícula contratada (estudiantes atendidos con recursos públicos en colegios privados) y 1.889.000 estudiantes en colegios privados (El Tiempo.Com, 20 de abril de 2017). Con algo más de 320.000 maestros del sector oficial, y de cientos de miles en el sector privado. La 
educación entendida como derecho fundamental sufre una afectación por situaciones estructurales o hechos coyunturales de violencia o acoso escolar que de igual manera transgreden el ejercicio laboral docente y la libertad de cátedra, la libre expresión, la seguridad personal y las posibilidades de éxito académico de los estudiantes. El acoso y la violencia escolar afectan no solo el derecho a la educación sino también otros derechos de carácter fundamental como bien lo reconoce el jurista José Alejandro Ruiz Forero con fundamento en el estudio que hace sobre el marco legal y jurisprudencial del acoso escolar en el país (2016, p. 51).

La Encuesta de Convivencia Escolar y Circunstancias que la Afectan (ECECA), para estudiantes de $5 .^{\circ}$ a $11 .^{\circ}$ de la Ciudad de Bogotá, realizada conjuntamente entre el Departamento Administrativo Nacional de Estadística (DANE) y la Secretaría de Educación de Bogotá, arroja unas cifras alarmantes ${ }^{1}$. Entre otros datos significativos, resulta conveniente destacar los siguientes:

$17,4 \%$ de los estudiantes de grado $5 .^{\circ}, 12,6 \%$ de los grados $6 .^{\circ}$ a $9 .^{\circ} \mathrm{y}$ $5,5 \%$ de los grados $10 .^{\circ}$ y $11 . .^{\circ}$ aseguran que un compañero de su curso los ofendió o les pegó todo el tiempo, durante el periodo de referencia. Por sexo se observa que los hombres están más expuestos a este tipo de situación (13,7\%), en comparación con las mujeres (9,8\%). Este tipo de comportamiento es muy similar si se compara por tipo de jornada y sector, aunque sobresale la jornada de la tarde con el 12,6\% y el sector oficial con el $12,2 \%$.

$11,4 \%$ de los estudiantes de grado $6 .^{\circ}$ a $9 .^{\circ}$ manifiestan que durante el periodo de referencia han sido víctimas de algún tipo de amenaza, ofen-

1 La recolección de la información se realizó entre el 1 de agosto y el 15 de septiembre del año 2011, en la ciudad de Bogotá, a excepción de la localidad de Sumapaz. El objetivo de la encuesta es identificar los factores que afectan la convivencia escolar en estudiantes de grados $5 .^{\circ}$ a $11 .^{\circ}$ de Bogotá, que se encuentran matriculados en establecimientos públicos y privados. De la misma manera, busca diagnosticar las percepciones y expectativas de las niñas, niños y adolescentes, con el fin de diseñar políticas que permitan mejorar la convivencia en el entorno escolar. Existen tres tipos de factores que pueden afectar la convivencia escolar. El primero son las características propias del estudiante. El segundo factor tiene que ver con las condiciones particulares del establecimiento educativo al que asiste y por último, el entorno familiar y barrial que lo acompaña. Siguiendo este análisis, a continuación se presentan los resultados más importantes de la encuesta, iniciando con la estructura y caracterización de la población de estudio. Se continúa con las circunstancias que podrían afectar el clima escolar y que se encuentran estrechamente relacionadas con el colegio. Finalmente se exponen los factores asociados al entorno barrial que podrían incidir directamente en la convivencia escolar (DANE, 2012, p. 1). 
sa o presión por parte de alguna persona del colegio, a través de internet. Por sexo se observa que las mujeres están más expuestas a este tipo de situación $(11,7 \%)$, en comparación con los hombres (9,6\%). Por tipo de jornada y sector se observa un comportamiento muy similar, aunque sobresale la jornada de la tarde con 11,4\% y el sector oficial con $11,0 \%$.

$29,5 \%$ de los estudiantes de los grados $5 .^{\circ}$ a $11 .^{\circ}$ de la ciudad de Bogotá, que se encuentran matriculados en establecimientos educativos oficiales, manifiestan que durante el periodo de referencia han visto « 5 o más veces», a alguna persona ocasionando daños de manera intencional en una propiedad del colegio. En los colegios privados, 17,3\% reportó esta misma situación.

$11,1 \%$ de los estudiantes de básica secundaria (entre los grados $6 .^{\circ}$ y $9 .^{\circ}$ ) y $9,5 \%$ de los estudiantes de media (entre los grados $10 .^{\circ}$ y $11 .^{\circ}$ ) aseguran haber pertenecido alguna vez a una pandilla. Por sexo, la proporción de estudiantes que han pertenecido a pandillas es superior en los hombres $(13,8 \%)$ que en la mujeres $(6,4 \%)$. De acuerdo con los resultados desagregados por tipo de jornada y sector, se observa una mayor participación en la jornada completa $(12,9 \%)$ y en el sector oficial $(12,0 \%)$.

Ambiente del aula: $84,5 \%$ de los estudiantes de grado $5 .^{\circ}$ opinan que la situación que más afecta el ambiente del salón de clase es la indisciplina. Los estudiantes de los grados $6 .^{\circ}$ a $9 .^{\circ}$ resaltan la indisciplina $(86,6 \%)$, sin embargo $56,7 \%$ opinan también que las agresiones verbales entre compañeros perturban el ambiente del salón de clase. Los estudiantes de los grados $10 .^{\circ}$ y $11 .^{\circ}$ resaltan la indisciplina $(82,1 \%)$, las agresiones verbales entre compañeros $(54,5 \%)$ y los chismes $(38,1 \%)$ como los principales eventos que inciden en el clima del aula.

$37,2 \%$ de los estudiantes de $5 .^{\circ}$ a $11 .^{\circ}$ de los establecimientos educativos oficiales de la ciudad de Bogotá informaron que algún compañero de su curso llevó armas blancas al colegio durante el periodo de referencia (DANE, 2012, pp. 3 a 12).

La creación del Sistema Nacional de Convivencia Escolar contó con un escenario académico y político propicio; el proceso legislativo que culmina con la expedición y posterior sanción presidencial de la Ley 1620 de 2013 se da en un marco de discusión y de propuestas que exigían acciones concretas del Estado para atender la aludida problemática del sector educativo. Importante reconocer que la jurisprudencia de la Corte Constitucional allanó el camino para el trámite y aprobación de esta ley; las sugerencias y órdenes que la corporación expresó en varias sentencias (Sentencia T-917 de 2006 y Sentencia T-905 de 
2011, por ejemplo) fueron un acicate para que la Ley 1620 fuese una realidad y posteriormente se dinamizara su implementación desde los cuestionamientos que la aludida Corte hace sobre la eficacia del Sistema Nacional de Convivencia Escolar en la Sentencia T-478 de 2015, cuya magistrada ponente fue Gloria Stella Ortiz Delgado y en la cual se trató el caso de Sergio Urrego, joven que se suicidó como resultado de una situación de acoso escolar, vulneración de derechos fundamentales y violación del debido proceso por parte de la institución educativa donde estudiaba (Ruiz Forero, 2016, pp. 53, 56 y 71). Otros países de la región, en la misma época, desplegaban iniciativas institucionales serias en la materia; casos de Perú (Congreso de la República, 2011, Ley nro. 29719) y Argentina (Congreso Argentino, 2013, Ley nro. 26.892). En España se le dio vida al Observatorio Estatal de Convivencia escolar en 2007 mediante el Real Decreto 275/2007, del 23 de febrero, que complementaba otras acciones a nivel de la nación y la ejecución de medidas equivalentes en el conjunto de las regiones (Cerezo, 2008, p. 333).

Inicialmente en el artículo se muestra de manera sucinta la normatividad de carácter internacional que obliga a Colombia en materia de garantía de los derechos de niños, niñas y adolescentes; seguidamente se presentan algunas normas nacionales que tiene que ver con el asunto en cuestión, y se avanza en destacar los elementos o dispositivos establecidos por la Ley 1620 de 2013 y finalmente se presentan algunas consideraciones sobre el proceso de implementación de la ley, su recibimiento e incidencia en las instituciones educativas desde la experiencia pedagógica de docentes en ejercicio.

\section{PROBLEMA DE INVESTIGACIÓN Y ESTRATEGIA METODOLÓGICA UTILIZADA}

A continuación se expone el problema de investigación, desde la formulación de la pregunta problematizadora y los referentes de orden metodológico; en fin, los elementos básicos que orientaron la ejecución de la investigación de la cual es producto el presente artículo.

A través de un interrogante se pretendió darle forma al problema de investigación, que ciertamente orientó las indagaciones, interpretaciones, reflexiones y constataciones que se hicieron necesarias en el referido ejercicio académico. Un problema de investigación que se plantea en los siguientes términos: ¿Qué elementos, instrumentos o mecanismos se introducen o innovan con la Ley 1620 de 2013 en materia de prevención y atención del acoso y de las diversas formas de violencia que afectan los ambientes escolares y los derechos humanos, sexuales y reproductivos de niños, niñas y adolescentes en el contexto socio-educativo colombiano? 
El estudio, del que es producto este artículo, se realizó desde unas particulares consideraciones y referentes metodológicos. Considerando las singularidades del objeto de estudio se asume el trabajo de análisis desde un enfoque socio jurídico e interdisciplinario que permitiera valorar los alcances y posibilidades de los desarrollos jurídicos y de las respectivas políticas públicas educativas en el propio terreno de los establecimientos educativos. Metodológicamente, es una investigación realizada desde un enfoque cualitativo, de carácter descriptivo y explicativo, a partir de fuentes primarias y secundarias, que requirió el empleo de la inducción, la deducción, el análisis, la hermenéutica y la síntesis, como métodos de estudio de la realidad; de otra parte, el análisis documental y de texto, la entrevista semiestructurada y el grupo focal permitieron el acopio de información.

En el artículo titulado Violencias, segregación en la escuela y derecho a la educación: acercamiento conceptual y a su realidad en Colombia (Bocanegra, 2017, pp. 99-129), publicado anteriormente, pudimos hacer unas precisiones de orden conceptual que revisten utilidad al momento de abordar la realidad del acoso y las violencias que afectan las instituciones educativas, las políticas públicas y reformas legales correspondientes. Importante diferenciar cada una de las categorías con las que se aborda la referida problemática socioeducativa, pero especialmente diferenciar cuándo se puede estar en una situación de violencia escolar, violencia en la escuela o violencia contra la escuela (Bocanegra, 2017, p. 112-113). El referido artículo constituye una síntesis relativamente útil.

\section{RESULTADOS}

\subsection{La protección de los derechos de los niños niñas y adolescentes como un imperativo en el derecho internacional}

Toda actuación de los estados y gobiernos en materia de derechos, garantías y libertades de los niños, niñas y adolescentes deberá cumplir con unos estándares y protocolos definidos por el derecho internacional en el marco de los tratados y convenios ratificados o suscritos por los países, realidad que los obliga a propiciar las condiciones materiales para que los derechos humanos tengan vigencia en sus territorios, brindando plenas garantías a todos sus habitantes, pero especialmente a los sectores que pueden afrontar situaciones de vulnerabilidad: discapacitados, minorías étnicas, infantes y jóvenes, entre otros. La comunidad internacional ha mostrado un interés constante por erradicar las formas de violencia que afectan a la niñez y la juventud; es así, que ha creado una serie de instrumentos jurídicos internacionales que buscan trascender la normativa interna de las naciones e instalar unos valores 
y dispositivos jurídicos supranacionales desde los cuales se asegure el reconocimiento del niño, niña y adolescente como sujetos de derecho.

Los estados nacionales modernos ya no tienen esa soberanía exclusiva para justificar la forma como tratan a las personas sometidas a su jurisdicción, muchas veces desconociendo y vulnerando derechos inherentes a ellas, sin el control y el juicio de reproche de nadie. La globalización del derecho ha abierto las puertas hacia el mundo exterior. Las constituciones ya no son documentos cerrados, en donde el tenor literal y la interpretación de la norma están exclusivamente ceñidos a lo escrito en ellas; ahora, con la integración del bloque de constitucionalidad, la Ley de leyes de nuestro ordenamiento jurídico (Constitución Política de 1991) no sólo se compone por las disposiciones contenidas en ella sino también por otras muchas que encontramos en los tratados y convenios internacionales de derechos humanos y de Derecho Internacional Humanitario (ICBF, 2009, p. 10).

La lucha contra la violencia escolar cuenta con los referentes jurídicos internacionales necesarios, y estos, como tales, han servido como estímulo para el impulso de políticas nacionales en un marco de cumplimiento de las obligaciones adquiridas en tratados, convenios y protocolos firmados y ratificados por las respectivas autoridades nacionales. Con el fin de salvaguardar los derechos de los niños, niñas y adolescentes, quienes pueden ser objeto de mayores niveles de victimización en el contexto de las diversas formas de violencia en la familia, los entornos barriales y la escuela, el derecho internacional ha alcanzado unos importantes desarrollos de un alto valor y alcance social. Vale la pena destacar alguna normatividad relevante en este sentido.

En la Declaración de Ginebra sobre los Derechos del niño (1924) se reconoce por primera vez la existencia de derechos determinados para los niños y niñas, teniendo el propósito de resguardar sus derechos fundamentales a la dignidad, la libertad de expresión, a no ser sujetos a tratos degradantes y no ser discriminados por razones de condiciones étnicas, sexo, idioma, opinión política o circunstancia de otra naturaleza. Esta declaración atribuye la obligación a los estados parte de garantizar todas las medidas administrativas y legislativas adecuadas para que el niño sea protegido contra toda forma de discriminación o punición arbitraria (artículo $2 .^{\circ}$ ).

En la misma dirección la Declaración de los Derechos del Niño (1959) instituye que los Estados deberán ofrecer amparo para el desarrollo físico, mental y social del niño en forma saludable y normal, así como en condiciones de libertad y dignidad. De igual manera, establece que todo niño tiene derecho a una edu- 
cación y a un tratamiento especial cuando afronta alguna discapacidad mental o física.

El Pacto Internacional de Derechos Económicos, Sociales y Culturales (1966) prevé que «se deben adoptar medidas especiales de protección y asistencia a favor de todos los niños y adolescentes, sin discriminación alguna por razón de filiación o cualquier otra condición» (artículo 10). Por su parte, la Convención Americana sobre Derechos Humanos o Pacto de San José de Costa Rica (1969) entroniza el principio de la corresponsabilidad (Estado, familia y sociedad) en la protección de los menores de edad; este principio es incorporado con mayor claridad al ordenamiento jurídico interno colombiano con la Ley 1098 de 2006, mediante la cual se expide el Código de Infancia y Adolescencia (Colombia. Congreso de la República, 2006).

Colombia, mediante la Ley 12 de 1991, acoge la Convención Internacional sobre los derechos del niño adoptada por la Asamblea General de las Naciones Unidas el 20 de noviembre de 1989. Como norma de carácter supranacional estipula de manera expresa la obligación por parte del Estado colombiano de brindar protección a los niños, niñas y adolescentes contra todo tipo de violencia que pueda presentarse en cualquier espacio de la vida social, familiar o institucional. Confirma la necesidad de proveer atención y asistencias especiales en razón de su vulnerabilidad, que se definan los instrumentos o mecanismos de orden institucional que garanticen la protección jurídica del infante, en el marco de los derechos, garantías y libertades que esta misma convención les reconoce.

De hecho, nos encontramos con una variada gama de recursos jurídicos de carácter supranacional que establece una variada escala de obligaciones que los estados deben asumir para prevenir y atender hechos o situaciones que afecten los derechos de niños, niñas y adolescentes desde una clara prohibición legal de toda forma de daño físico o mental, pero igualmente asegurando acciones que promuevan la convivencia como condición para la propia vigencia de los derechos que le asisten a cada cual, especialmente en el contexto de los establecimientos escolares ${ }^{2}$. La Convención Internacional sobre los derechos del niño ratifica el criterio y necesidad de no establecer excepción alguna en materia de garantía de los derechos a los niños y niñas, la obligación que le corresponde al Estado de desplegar toda su capacidad en la dirección de protegerlos de toda forma de discriminación o violencia.

2 Organización de la Naciones Unidas. Comité de los Derechos del Niño. 18 de abril de 2011. Observación General nro. 13 sobre el derecho del niño a no ser objeto de ninguna forma de violencia. CRC/C/GC/13. 


\subsection{Marco jurídico en Colombia contra las violencias y el acoso escolar}

Con la Constitución Política de 1991 se pretendió robustecer las garantías constitucionales de los niños, niñas y adolescentes como sujetos de especial protección y darle materialidad institucional a los lineamientos jurídicos internacionales a que se obligó el Estado colombiano con la firma, ratificación o aceptación de numerosos tratados, convenios y recomendaciones en materia de derechos humanos.

Según el criterio de la Corte Constitucional (Sentencia C-574 de 1992), con los artículos 93, 94 y 214 de la Constitución Nacional se le ha conferido a esa normatividad humanitaria un rango supraconstitucional, de modo que opera una incorporación automática de la misma en el ordenamiento interno colombiano (ICBF, 2009, p. 11).

Por su parte el artículo 44 de la Constitución Política de 1991 es claro al establecer un cúmulo bien importante de derechos fundamentales que le son propios a los niños y niñas; «consagra la obligación de la familia, la sociedad y el Estado de asistir y proteger al niño para su desarrollo armónico e integral».

Conviene destacar una realidad que salta a la vista. Después de 22 años de aprobada la Carta Política de 1991, la Ley 1620 de 2013 crea el Sistema Nacional de Convivencia escolar y con él se instituye un andamiaje institucional para atender una problemática que compromete el futuro y posibilidad de la educación en el país y de millones de niños, niñas y adolescentes integrados en miles de instituciones educativas a todo lo largo y ancho del territorio nacional. Como se expresaba anteriormente, la aprobación de esta ley contó con un contexto social, académico y político relativamente favorable; el despliegue mediático, los trabajos académicos y los pronunciamientos de colectivos de derechos humanos contribuyeron a crear un clima favorable a la referida iniciativa, pero después de que en el Congreso de la República fracasaran 27 iniciativas legislativas con las que se pretendía establecer una normatividad para la prevención y corrección de las diversas modalidades de violencia escolar, según se argumentó en la exposición de motivos que soportó el debate legislativo que precedió la aprobación de la referida ley (Colombia. Congreso de la República. Proyecto de Ley 201 de 2012, Cámara de Representantes, p. 16).

De todos modos, no se puede negar que la violencia y el acoso escolar, la vulneración de los derechos de niños, niñas y adolescentes en el ejercicio cotidiano de la vida escolar es una cosa de vieja data; seguramente los episodios de violencia escolar aunque igualmente numerosos y graves como los que se presentan en la actualidad, no revistieron, en décadas pasadas, la atención 
mediática, académica o política adecuada que exigiera el diseño de unos instrumentos jurídicos y de política pública para atender tal problemática. La violencia y el acoso escolar fueron una realidad que le correspondió afrontar a generaciones anteriores, con todo y las implicaciones culturales, sociales y psicológicas que esta situación pudo tener. En 1992 el trabajo académico publicado con el título de La escuela violenta da cuenta de la aludida situación en el país (Parra Sandoval, González, Moritz, Blandón, Bustamante, 1992).

\subsection{El valor de la Ley 115 de 1994 en materia convivencial y formación ciudadana}

El primer impulso normativo interno para atender la convivencia escolar lo constituye la Ley 115 de 1994 o Ley General de Educación. Esta norma, producto de un amplio proceso de concertación en el cual intervinieron varios actores vinculados o relacionados con el proceso educativo, marcó unos derroteros claves para el sector en materia de organización del servicio, autonomía curricular, democracia escolar, fines y objetivos de la educación, la educación para grupos étnicos, adultos, campesinos y personas discapacitadas. En su artículo $1 .^{\circ}$ esta ley establece:

De conformidad con el artículo 67 de la Constitución Política, define y desarrolla la organización y la prestación de la educación formal en sus niveles preescolar, básica (primaria y secundaria) y media, no formal e informal, dirigida a niños y jóvenes en edad escolar, a adultos, a campesinos, a grupos étnicos, a personas con limitaciones físicas, sensoriales y psíquicas, con capacidades excepcionales, y a personas que requieran rehabilitación social (Colombia. Congreso de la República, Ley 115 de 1994).

La Ley 115 acentúa la convivencia como componente curricular clave en la formación integral de los educandos desde el respeto a la vida, los derechos humanos, a la paz, a los principios democráticos de convivencia, pluralismo, justicia, solidaridad, equidad y el ejercicio de la tolerancia y la libertad (artículo $\left.5 .^{\circ}\right)$ y el fomento en la institución educativa de prácticas democráticas para el aprendizaje de los principios y valores de la participación y organización ciudadana que estimulen la autonomía y la responsabilidad (literal c, artículo 13), así como proporcionar una sólida formación ética y moral, y fomentar la práctica y el respeto por los derechos humanos (literal b, artículo 13).

Pero en términos prácticos se destaca de la aludida ley una disposición bien importante en materia de formación ciudadana: la obligatoriedad de todos los establecimientos oficiales o privados de educación formal en los niveles de la educación preescolar, básica y media, de la enseñanza de contenidos que 
promuevan el estudio, la comprensión y la práctica de la Constitución y la instrucción cívica; y la educación para la justicia, la paz, la democracia, la solidaridad, la confraternidad, el cooperativismo y la formación en los valores humanos (artículo 14).

Con esta norma es notorio el avance en materia de participación y garantías democráticas para los estamentos de las comunidades educativas. A diferencia de la estructura de gobierno escolar anterior, la Ley 115 de 1994 estableció órganos de dirección colegiada (Consejo directivo y Consejo académico) integrados por representantes de los estamentos básicos escolares: profesores, estudiantes, padres de familia, egresados y sector productivo. Todos ellos participarán en el diseño, ejecución y evaluación del Proyecto Educativo Institucional.

Un ambiente de mayor democracia escolar contribuyó en su momento a dinamizar los procesos pedagógicos y asegurar una convivencia escolar favorable al debate académico y las buenas prácticas sociales en la escuela. De los procesos de conformación del gobierno escolar, elección del personero estudiantil y los representantes de grado al consejo de estudiantes se forjaron importantes liderazgos que trascendieron los muros de los colegios, según lo enseña la experiencia de egresados de colegios públicos de la ciudad de Bogotá que atendieron una convocatoria para trabajar en grupo focal en el mes de agosto de 2016.

\subsubsection{El Decreto 1860 de 1994, Manual de Convivencia y Proyecto Educativo Institucional}

Los lineamientos generales contenidos en la Ley 115 de 1994 fueron reglamentados por el Decreto 1850 de 1994, norma que especifica los aspectos pedagógicos y organizativos del servicio educativo, del Proyecto Educativo Institucional y del manual de convivencia. Quedaron establecidos los requisitos que en materia convivencial deben considerar las instituciones educativas en el proceso de adopción de los manuales de convivencia. La obligación de tener en cuenta los principios constitucionales que deben regir la comunidad educativa, la organización respecto a la convivencia escolar, normas de conducta de los estudiantes y docentes basadas en el mutuo respeto y los procedimientos para resolver los conflictos individuales y colectivos entre miembros de la comunidad educativa y el sistema de evaluación del desempeño escolar (artículo 17).

Tanto la Ley 115 de 1994 como su decreto reglamentario definieron durante dos décadas el rumbo académico y convivencial de las instituciones educativas de preescolar, básica y media en Colombia. Corresponderá hacer 
un balance mucho más profundo sobre los alcances de estas disposiciones normativas en la calidad y pertinencia de la educación y la democracia escolar.

\subsection{El sistema nacional de convivencia escolar y formación para el ejercicio de los derechos humanos, la educación para la sexualidad y la prevención y mitigación de la violencia escolar}

Una vez surtido el respectivo proceso legislativo en el Congreso de la República se sancionó la Ley 1620 de 2013, una iniciativa que tuvo como protagonista al Ministerio de Educación Nacional, los representantes a la Cámara Juana Carolina Londoño, Simón Gaviria Muñoz y Telésforo Pedraza; el apoyo de la Alta consejería para los derechos humanos, el ICBF, el Ministerio de las TIC y la Policía Nacional (Congreso de la República, Informe para primer debate al Proyecto de Ley 159 de 2012 Senado; 201 de 2012 Cámara de Representantes, 27 de noviembre de 2012).

Precedida de la respectiva argumentación jurídica, política y pedagógica, la iniciativa tenía el propósito de establecer una regulación jurídica que contribuyera al mejoramiento de la convivencia en los establecimientos educativos del país, pero que también los orientara respecto a la formación para el ejercicio de los derechos humanos, sexuales y reproductivos, su promoción, la prevención y mitigación de la violencia y el acoso escolar, todo ello conforme a la Constitución Política que reconoce a la educación como un derecho y que es responsabilidad del Estado, la sociedad y la familia (artículo 67). El objeto de la Ley 1620 de 2013 se define claramente en su artículo 1. ${ }^{\circ}$ :

contribuir a la formación de ciudadanos activos que aporten a la construcción de una sociedad democrática, participativa, pluralista e intercultural, mediante la creación del sistema nacional de convivencia escolar y formación para los derechos humanos, la educación para la sexualidad y la prevención y mitigación de la violencia escolar, que promueva y fortalezca la formación ciudadana y el ejercicio de los derechos humanos, sexuales y reproductivos de los estudiantes de preescolar, básica y media y prevenga y mitigue la violencia escolar y el embarazo en la adolescencia (Colombia. Congreso de la República, 2016).

De entrada la ley hace unas precisiones de orden conceptual, que en términos de la pedagogía de la misma norma resultan pertinentes y útiles: competencias ciudadanas, acoso escolar o bullying; cyberbullying o ciberacoso (artículo 2. ${ }^{\circ}$ ). En el Decreto Reglamentario 1965 de 2013 se amplía el ejercicio conceptual incorporando otras definiciones necesarias para el ejercicio de interpretación y aplicación normativa; tenemos: conflictos, conflictos 
manejados inadecuadamente, agresión escolar, agresión física, agresión gestual, agresión relacional, agresión electrónica, violencia sexual, vulneración de los derechos, restablecimiento de los derechos de niños, niñas y adolescentes (Colombia. Ministerio de Educación Nacional, 2016).

\subsubsection{Creación del Sistema Nacional de Convivencia Escolar}

Esta normatividad creó el Sistema Nacional de Convivencia Escolar y Formación para el Ejercicio de los Derechos Humanos, la Educación para la Sexualidad y la Prevención y Mitigación de la Violencia Escolar, en la perspectiva de garantizar la protección de los educandos contra las diversas formas de violencia y acoso escolar que cotidianamente se denunciaban; garantizar la existencia de una ruta de atención integral para los conflictos escolares; fomentar y fortalecer la educación en y para la paz; y garantizar los mecanismos de prevención, protección, detección temprana y denuncia de todas aquellas conductas que atenten contra la convivencia y los derechos de los miembros de la comunidad educativa, especialmente de los educandos.

La aludida ley obliga a crear comités de convivencia escolar en todos los niveles (nacional, departamental, municipal y en las instituciones educativas) y definir una ruta de atención integral para la convivencia escolar y el funcionamiento de un sistema de información unificado de convivencia escolar para la toma de decisiones en cada nivel territorial, además de ordenar la aplicación efectiva de los manuales de convivencia. Como un antecedente de estos comités en Bogotá en el año 2000, el Consejo de la ciudad (Acuerdo 04 de 2000) creó los comités de convivencia de las instituciones educativas públicas y privadas del Distrito Capital; un órgano integrado por representantes de los distintos estamentos de la comunidad educativa y presidido por el rector o un coordinador. Estos organismos funcionaron hasta la promulgación de la Ley 1620.

\subsubsection{Objetivos y principios del Sistema Nacional de Convivencia Escolar}

Entre los objetivos más destacados de la Ley 1620 tenemos: el fortalecimiento de la convivencia escolar para el ejercicio de los derechos humanos, sexuales y reproductivos de los niños, niñas y adolescentes; la garantía de la protección integral de la niñez y adolescencia en los espacios educativos, así como el fomento de derechos y principios constitucionales para la formación de sujetos activos de derechos; la promoción y el fomento a los mecanismos para la prevención, detención, atención y seguimiento de los casos de violencia escolar, acoso escolar o vulneración de derechos sexuales y reproductivos. Por último, el sistema debe orientar estrategias y programas de comunicación relacionados con la convivencia escolar, la construcción de ciudadanía y la promoción 
de los derechos humanos, sexuales y reproductivos, especialmente lo referido a la prevención de embarazo en adolescencia y el mejoramiento del clima escolar (Congreso de la República, Ley 1620 de 2013, artículo 4..$^{\circ}$.

Varios principios orientan el Sistema Nacional de Convivencia Escolar, tenemos el de participación, que está referido, de una parte, a la coordinación y armonización de acciones entre las diferentes entidades públicas responsables, de conformidad con la Ley 115 de 1994, la Ley 1098 de 2006 y la Constitución Política; de otra, a la participación activa de niños, niñas y jóvenes en el desarrollo de las distintas acciones del sistema; el principio de la corresponsabilidad que orienta el papel de instituciones educativas, la familia el Estado y la sociedad en el cumplimiento de los objetivos del sistema; y los principios de autonomía, diversidad e integralidad (Congreso de la República, Ley 1620 de 2013, artículo 5. ${ }^{\circ}$.

El sistema se organiza en tres niveles. El primero es el nivel nacional conformado por el Comité Nacional de Convivencia Escolar, el cual estará integrado de manera permanente por los ministros de Educación Nacional, de Salud, de Cultura y de las TIC así como el Defensor del Pueblo y el director de la Policía Nacional, el director del Instituto Colombiano de Bienestar familiar, el responsable del Sistema de Responsabilidad Penal de Adolescentes y el presidente de la Asociación Colombiana de Facultades de Educación, el presidente de la Asociación Colombiana de Escuelas Normales Superiores, entre otros. Teniendo como principales funciones: definir la operación del Sistema en cada uno de los niveles o instancias, coordinar la gestión, armonizar las acciones de funcionamiento, comunicación y vigilancia del Sistema, y garantizar la adopción de la Ruta de Atención Integral para la Convivencia Escolar. Se percibe un esfuerzo por articular distintas instituciones del Estado en apoyo de un idéntico y único propósito, definiendo «quienes serán los encargados de materializar los objetivos trazados» (Molina Peláez, 2017, p. 77).

El segundo nivel está representado por los comités departamentales, distritales y municipales de convivencia escolar, integrados por los secretarios de gobierno, educación, salud y cultura y por los representantes regionales o locales de las mismas entidades o autoridades de los mismos sectores que hacen presencia en el comité nacional. Las principales funciones de estos comités son las de armonizar, articular y coordinar las acciones del sistema en la jurisdicción; garantizar la Ruta de Atención; el fomento de competencias y proyectos pedagógicos para promover el ejercicio de derechos humanos, sexuales y reproductivos; el registro oportuno y confiable de la información correspondiente; recomendar, vigilar, revisar y ajustar las estrategias y acciones del sistema en la respectiva jurisdicción (Congreso de la República, Ley 1620 de 2013, artículos $5 .^{\circ}$ a $\left.10 .^{\circ}\right)$. 
El tercer nivel lo representa el comité escolar de convivencia, célula básica del sistema; el de mayor valor dada su relación directa con la problemática social y convivencial que afecta a los establecimientost educativos. Integrado por el rector, personero estudiantil, representantes de los docentes y coordinadores del establecimiento educativo, así como los presidentes del consejo de padres y de estudiantes. Este comité se constituye en el principal responsable de las políticas institucionales en materia de convivencia escolar según las propias funciones que le asigna la ley. Entre ellas, las de identificar, documentar, analizar y resolver los conflictos que se presenten entre miembros de la comunidad educativa; liderar acciones que fomenten la convivencia, la construcción de ciudadanía, el ejercicio de los derechos humanos, sexuales y reproductivos, y la prevención y mitigación de la violencia escolar; convocar a un espacio de conciliación para la resolución de situaciones conflictivas que afecten la convivencia escolar; liderar el desarrollo de estrategias e instrumentos destinados a promover y evaluar la convivencia escolar; el ejercicio de los derechos humanos sexuales y reproductivos; hacer seguimiento al cumplimiento de las disposiciones establecidas y presentar informes a la respectiva instancia que hace parte de la estructura del sistema (Congreso de la República, Ley 1620 de 2013, artículo 13).

Los comités escolares de convivencia están obligados a seguir los lineamientos fijados en la Ruta de Atención para la Convivencia Escolar, desarrollados en el capítulo V de la ley (Herramientas del Sistema), cuando dada la naturaleza y alcance de los hechos, conflicto o situación de violencia o vulneración de derechos humanos, sexuales y reproductivos, no pueda ser asumido o decidido por este organismo al trascender el ámbito pedagógico y se constituyan en hechos o conductas punibles que deben ser avocados por otras autoridades competentes, judiciales y administrativas que la misma norma integra al sistema, por ejemplo la Fiscalía General de la Nación, el Sistema de Responsabilidad Penal de Adolescentes o el Instituto Colombiano de Bienestar Familiar, según sea el caso (Ley 1620 de 2013. Artículo 13, numeral 5. ${ }^{\circ}$ ).

Finalmente, desde la consideración pedagógica de que la convivencia escolar y la formación ciudadana son asuntos de naturaleza curricular, este comité debe proponer y analizar estrategias de flexibilización del modelo pedagógico y articular las diferentes áreas del plan de estudios (Ley 1620 de 2013, artículo 13 , numeral $8 .^{\circ}$ ); en fin, le corresponde estructurar una propuesta pedagógica, didáctica y metodológica que permita las mejores condiciones de aprendizaje, motivación y desempeño de los educandos.

El anterior criterio tiene un valor significativo de cara a crear condiciones para una sana convivencia en las instituciones educativas y unos procesos coherentes y pertinentes en materia de formación ciudadana. En un colegio los aspectos 
académicos y convivenciales perviven, se nutren mutuamente, se construyen en un proceso en el cual las lógicas y discursos científicos contribuyen a la formación convivencial del estudiante, pero igualmente donde el acatamiento o respeto de este por los principios éticos y jurídicos escolares es una condición necesaria para avanzar en el trabajo de aula y con ello lograr una mejor apropiación, asimilación o conocimiento del mundo de la ciencia, el arte y la tecnología.

\subsubsection{El Sector Educativo en el Sistema Nacional de Convivencia Escolar y Formación para los Derechos Humanos, la Educación para la Sexualidad y la Prevención y Mitigación de la Violencia Escolar}

Como resulta obvio, por el objeto de la ley que creó el referido sistema, el sector educativo se constituye en su piedra angular. Este se integra por el Ministerio de Educación Nacional, las secretarías de educación de las entidades territoriales certificadas en educación y los establecimientos que prestan el servicio educativo de acuerdo con la Ley 115 de 1994; adicionalmente, es importante reconocer que la Ley 1620 de 2013 establece un conjunto de responsabilidades que le corresponde asumir a cada una de estas entidades frente a los derechos humanos, la educación para la sexualidad y la prevención y mitigación de la violencia escolar (capítulo III, artículos 14 a 22).

En el marco del referido sistema, al Ministerio de Educación Nacional, por ejemplo, se le asignaron funciones relacionadas con la promoción y el fomento, la coordinación interinstitucional, divulgación, asistencia técnica, diseño y administración del Sistema de Información Unificado de Convivencia Escolar (Ley 1620 de 2013, artículo 15).

De la misma manera, a las entidades territoriales se les asignaron funciones primordialmente de ejecución y coordinación, pero igualmente otras relacionadas con la armonización institucional, divulgación, formación docente, acompañamiento institucional a los colegios, de seguimiento y apoyo a los casos de acoso escolar reportados y la garantía de implementación de la Ruta de Atención Integral para la Convivencia Escolar en todos los establecimientos educativos (Ley 1620 de 2013, artículo 16).

Finalmente, la lista de responsabilidades de los establecimientos educativos es alargada por la Ley 1620 de 2013, respecto a lo instituido por otras normas en materia educativa: Ley 115 de 1994 y Decreto Reglamentario 1860 de 1994 (el Decreto Reglamentario 1860 fue recogido en el Decreto 1075 del 26 de mayo de 2015, por medio del cual se expide el Decreto Único Reglamentario del Sector Educación). Igualmente otras normas como la Ley 1098 de 2006 o Código de Infancia y Adolescencia, que establece un cúmulo 
de obligaciones para los establecimientos educativos; la Ley 1098, que contiene cuatro acápites al respecto: el artículo 42 incorpora doce (12) Obligaciones especiales de las instituciones educativas; el artículo 43 define en tres (3) numerales la Obligación ética fundamental, y el artículo 44 incorpora diez (10) Obligaciones complementarias que deben asumir los establecimientos escolares; finalmente este Código establece la Prohibición de sanciones crueles, humillantes o degradantes:

Los directores y educadores de los centros públicos o privados de educación formal, no formal e informal, no podrán imponer sanciones que conlleven maltrato físico o psicológico de los estudiantes a su cargo, o adoptar medidas que de alguna manera afecten su dignidad. Así mismo, queda prohibida su inclusión bajo cualquier modalidad, en los manuales de convivencia escolar (Colombia. Congreso de la República, Ley 1098 de 2006, artículo 45).

La Ley 1620 de 2013 clarifica las responsabilidades de los rectores y docentes de los establecimientos educativos (artículos 18 y 19) en el Sistema Nacional de Convivencia Escolar. Precisamente en el marco de responsabilidades de los docentes se destaca un asunto que puede tener trascendencia en el devenir convivencial y académico de los establecimientos escolares: la transformación de las prácticas pedagógicas para la construcción de ambientes democráticos y tolerantes (numeral 1 del artículo 19); igualmente resulta destacable el planteamiento de que los proyectos pedagógicos convivenciales, además de ser una construcción colectiva para todos los grados, deberán ser formulados y ejecutados por los docentes de todas las áreas. Es decir, la formación para la convivencia y el respeto de los derechos de los demás es un asunto que involucra a todo el personal docente, independiente de la asignatura que regente. No son los directores de grupo, orientadores escolares o coordinadores de disciplina los únicos responsables de la promoción de la sana convivencia; se requiere que el referido asunto sea un elemento trasversal del currículo y un compromiso de todos los actores del proceso educativo.

Sobre los manuales de convivencia, herramienta fundamental de regulación escolar, y en el marco de la creación del Sistema Nacional de Convivencia Escolar y Formación para los Derechos Humanos, la Educación para la Sexualidad y la Prevención y Mitigación de la Violencia Escolar, se amplía lo establecido en la Ley 115 de 1994 (Ley General de Educación) sobre la materia y se establecen unas disposiciones que tienen un valor práctico: permanente actualización en la perspectiva de identificar nuevas formas y alternativas para incentivar y fortalecer la convivencia escolar y el ejercicio de los derechos humanos, sexuales y reproductivos de los estudiantes; en esa dirección se le otorga al docente la carga de obrar como un orientador y mediador en circunstancias 
que transgredan la convivencia y los derechos (Ley 1620 de 2013, artículo 21). El manual de convivencia debe definir los derechos y obligaciones de los estudiantes, garantizar el debido proceso e incluir la Ruta de Atención Integral, y se ratifica el criterio de que en su elaboración o reforma se debe garantizar la participación de los estudiantes, los padres de familia y la comunidad educativa en general.

La Ley 1620 no deja de lado la participación de la familia (artículo 22); como actor clave de la comunidad educativa se le especifican unas obligaciones que deberá cumplir en el perspectiva de que los educandos cuenten con las condiciones para su formación académica, entre ellas «proveer a sus hijos espacios y ambientes en el hogar, que generen confianza, ternura, cuidado, y protección de sí y de su entorno físico, social y ambiental» (Colombia. Congreso de la República, Ley 1620 de 2013, artículo 22, numeral 1); cumplir con las condiciones del manual de convivencia (artículo 22, numeral 6); y conocer la Ruta de Atención Integral (artículo 22, numeral 7).

Conjuntamente con el sector educativo y las entidades públicas correspondientes de cada nivel político administrativo, otros sectores y entidades hacen parte del Sistema Nacional de Convivencia Escolar en lo de su competencia y en la perspectiva de que contribuyan a prevenir, resolver, atender o mitigar situaciones de violencia escolar. Son ellos, el Ministerio de Salud, el Ministerio de Cultura, el Instituto Colombiano de Bienestar Familiar, la Defensoría del Pueblo, las autoridades judiciales integrantes del Sistema de Responsabilidad Penal de Adolescentes, los personeros municipales o distritales en representación del Ministerio Público y la Policía de Infancia y Adolescencia que se constituye en el enlace con los rectores y directores de los establecimientos educativos. Una consideración corresponde hacer a propósito de esta realidad institucional que se crea. Primero, el evidente reconocimiento de que la violencia en los establecimientos educativos no es un asunto cuya responsabilidad deba quedar en los linderos de lo escolar o en cabeza exclusiva de las autoridades escolares, sino que es una problemática que trasciende el espacio institucional de la escuela; segundo, que la envergadura y complejidad del problema, la violencia escolar y la violencia en la escuela, hace necesario una mirada y atención interinstitucional.

\subsubsection{Las Herramientas del Sistema Nacional de Convivencia Escolar y formación para los derechos humanos, la educación para la sexualidad y la prevención y mitigación de la violencia escolar}

La toma de decisiones en cualquier asunto público y la definición de las políticas públicas que permitan atender situaciones de conflicto o resolver necesidades sociales deben estar precedidas de información fidedigna que las soporte. 
El Sistema de Información Unificado de Convivencia Escolar (artículo 28) es una respuesta a este imperativo social, político y administrativo. Se pretende contar con los instrumentos que permitan identificar, registrar y hacer seguimiento de los casos de acoso, violencia escolar y vulneración de derechos sexuales y reproductivos que afecten a los niños, niñas y adolescentes de los establecimientos educativos, en la perspectiva de que en cada nivel se definan las políticas, programas o acciones pertinentes en el marco de la Ruta de Atención Integral para la Convivencia Escolar que la misma Ley 1620 establece como otra herramienta del sistema (artículo 29); que a su vez contempla unos procesos y protocolos que en todos los casos que se vea vulnerada la convivencia escolar o los derechos humanos, sexuales y reproductivos de los estudiantes deben seguirse en los niveles de preescolar, básica y media, así como de casos de embarazo en adolescentes y que pueden requerir la intervención de autoridades externas al ámbito escolar: Fiscalía General de la Nación, Instituto Colombiano de Bienestar familiar-Defensoría de familia, Comisaría de Familia o Policía de Infancia y Adolescencia.

La Ruta de Atención Integral para la Convivencia Escolar se desarrolla desde cuatro componentes: promoción, prevención, atención y seguimiento. Cada una de las entidades que integran el sistema, especialmente los establecimientos educativos, deberá responder por el planeamiento, ejecución, control y evaluación de las estrategias o acciones que le den forma a cada uno de estos componentes siguiendo los postulados, procedimientos y protocolos que la misma ley establece o que las autoridades competentes definan en el marco de la competencia reglamentaria que tienen o que los colegios establezcan en el marco de las facultades que les concede para definir su Proyecto Educativo Institucional y su manual de convivencia.

De todos modos la Ley 1620 en su artículo 31 establece unos postulados básicos que deberán guiar la formalización de los protocolos y procedimientos de la Ruta de Atención Integral para la Convivencia Escolar. Estos postulados son: "la puesta en conocimiento de los hechos por parte de cualquier miembro de la institución... el conocimiento de estos hechos a los padres de familia o acudientes según el caso de las víctimas o de los generadores de los hechos violentos... se buscaran alternativas de solución... Se garantice la atención integral y el seguimiento pertinente para cada caso (Ley 1620 de 2013, artículo 31).

De otra parte, la norma establece tres criterios que son importantes a la hora de definir o darle forma a los protocolos y procedimientos referidos:

1. La Ruta de Atención Integral inicia con la identificación de situaciones que afectan la convivencia por acoso o violencia escolar, las cuales tendrán 
que ser remitidas al Comité Escolar de Convivencia, para su documentación, análisis y atención a partir de la aplicación del manual de convivencia.

2. El componente de atención de la ruta será activado por el Comité de Convivencia Escolar por la puesta en conocimiento por parte de la víctima, estudiantes, docentes, directivos docentes, padres de familia o acudientes, de oficio por el Comité de Convivencia Escolar o por cualquier persona que conozca de situaciones que afecten la convivencia escolar.

3. Una vez agotada esta instancia, las situaciones de alto riesgo de violencia escolar o vulneración de derechos sexuales y reproductivos de niños, niñas y adolescentes de los establecimientos educativos en los niveles de preescolar, básica y media que no puedan ser resueltas por las vías que establece el manual de convivencia y se requiera la intervención de otras entidades o instancias, serán trasladadas por el rector de la institución, de conformidad con las decisiones del Comité Escolar de Convivencia, al ICBF, la Comisaria de Familia, la Personería Municipal o Distrital o a la Policía de Infancia y Adolescencia, según corresponda (Ley 1620 de 2013, artículo 31).

Finalmente, la ley establece un acápite de infracciones administrativas, sanciones e incentivos. Siendo objeto de sanciones los actores que hacen parte del sistema por omisión, incumplimiento o retraso en la implementación de la Ruta de Atención Integral o en el funcionamiento del sistema en cada uno de sus niveles $\mathrm{u}$ organismos, conforme a lo determinado en el Código General y de Procedimiento Penal, el Código Único Disciplinario y el Código de la Infancia y la Adolescencia (Ley 1620 de 2013. Capítulo VI, artículos 35 a 39). La norma específica el tipo de infracciones y sanciones en que pueden incurrir las instituciones educativas privadas y hace una alusión a las faltas disciplinarias y sanciones de los docentes y directivos docentes de establecimientos educativos oficiales por omisiones o incumplimientos en el contexto de responsabilidades establecidas en el sistema.

Con la expedición del Decreto Reglamentario 1965 de 2013 el Gobierno Nacional reglamenta el funcionamiento del Sistema Nacional se Convivencia Escolar y Formación para los Derechos Humanos, la Educación para la Sexualidad y la Prevención y Mitigación de la Violencia Escolar. Este decreto reglamenta la organización, sesiones, quorum decisorio, actas, causales de impedimentos y recusaciones, mecanismos para la designación de algunos representantes, conformación de una mesa técnica y designación de la secretaría técnica del comité y define sus funciones. Hace lo propio con los comités municipales, distritales y departamentales de convivencia y con los comités 
escolares de convivencia. También crea y le da funciones a la Mesa Técnica del Sistema de Información Unificado de Convivencia Escolar, su operación y reporte de información.

En el título III del Decreto 1965 se establecen los Lineamientos generales para incorporar en el manual de convivencia de los establecimien tos educativos las disposiciones sobre manejo de situaciones que afectan la convivencia escolar, los derechos humanos, sexuales y reproductivos. Su artículo $28^{3}$ obliga a los colegios a incluir en los manuales de convivencia las definiciones, principios y responsabilidades que para todos los miembros de la comunidad educativa establece la Ley 1620 de 2013, para el desarrollo de los componentes de la Ruta de Atención Integral para la Convivencia Escolar, sin menoscabo de los demás aspectos que deben ser reglados en dichos manuales, en concordancia con lo determinado en la Ley 115 de 1994 y en el Decreto 1860 de 1994; el artículo 29 señala los lineamientos generales para su actualización ${ }^{4}$ y el artículo 30 define el plazo para que dicha actualización se haga (6 meses contados a partir de la publicación del Decreto 1965) 5 .

De manera complementaria, la referida norma (Decreto 1965) atiende lo correspondiente a las acciones que cada comité, en el nivel respectivo, deberá adelantar frente a cada uno de los componentes de la Ruta de Atención Integral; es decir, señala qué les corresponde hacer en materia de promoción, prevención, atención y seguimiento. Igualmente, clasifica las situaciones que afectan la convivencia escolar y el ejercicio de los derechos humanos, sexuales y reproductivos. Establece la finalidad, contenido y aplicación de los protocolos para atender cada una de estas situaciones según se presenten: Situaciones Tipo I, conflictos manejados inadecuadamente; Situaciones Tipo II, agresión escolar, acoso escolar (bullying) y ciberacoso (ciberbullying); Situaciones de tipo III, «situaciones de agresión escolar que sean constitutivas de presuntos delitos contra la libertad, integridad y formación sexual, referidos en el Título IV del Libro II de la Ley 599 de 2000, o cuando constituyen cualquier otro delito establecido en la ley penal colombiana vigente» (Colombia. Ministerio de Educación Nacional. Decreto 1965 de 2013, artículos 40 y 41).

3 Artículo compilado en el artículo 2.3.5.3.1 del Decreto Único Reglamentario 1075 de 2015.

4 Artículo compilado en el artículo 2.3.5.3.2 del Decreto Único Reglamentario 1075 de 2015.

5 Artículo compilado en el artículo 2.3.5.3.3 del Decreto Único Reglamentario 1075 de 2015. 


\section{LAS POSIBILIDADES Y LAS TALANQUERAS}

Conveniente valorar desde la experiencia de los docentes, directivos docentes e instituciones educativas el impacto o incidencia que ha tenido la Ley 1620 de 2013 sobre la vida escolar, en cotidianeidad del ejercicio pedagógico y sobre el ordenamiento y las prácticas institucionales de los diferentes actores que hacen parte del Sistema Nacional de Convivencia Escolar. Algunas experiencias y percepciones sobre el asunto podrían abrir el camino para una valoración mucho más extensa y rigurosa. Desde la experiencia de 10 docentes de Bogotá, 6 del sector educativo público y 4 del sector privado (entre los que se encontraban cuatro orientadoras escolares, dos coordinadores de convivencia y cuatro profesores de aula) de las localidades de Suba, Engativá y San Cristóbal se recogió información sobre sus experiencias en los procesos de ajuste del ordenamiento institucional convivencial exigido por la Ley 1620 de 2013 y su Decreto Reglamentario 1965 de 2013. La entrevista semiestructurada y dos grupos focales, uno integrado por docentes y directivos docentes y otro conformado por orientadoras escolares, que sesionaron durante el mes de noviembre de 2016, permitieron el acopio de información desde la cual se hicieron los respectivos planteamientos que se exponen en el presente escrito.

La Ley 1620 de 2013 pretendió dotar a las autoridades públicas, instituciones educativas públicas y privadas, directivos docentes y docentes, de las herramientas necesarias para enfrentar los actos de acoso y violencias que se presentan en el ámbito escolar. Sin embargo, tal tarea incumbe en igual medida a las familias de los estudiantes, pues solamente a partir del trabajo mancomunado entre estas y aquellas, es posible lograr una intervención efectiva sobre factores que pueden estar propiciando o favoreciendo situaciones de violencia donde los escolares actúan, ya como víctimas, ya como victimarios. Vincular a las familias a los procesos del colegio, que reconozcan los factores que desde el barrio, los grupos juveniles o la misma familia afectan la psiquis del niño, niña y adolescente y que además actúen positivamente sobre ellos, ha sido una verdadera proeza para los maestros. Nada más difícil que articular a las familias con la solución de los problemas escolares originados por las múltiples violencias que agobian a las instituciones educativas.

La regulación jurídica que se tiene en el país para afrontar el rigor de la violencia en los establecimientos educativos aporta en elementos conceptuales, herramientas y mecanismos para pensar o formular acciones para la promoción, prevención y atención convivencial, pero las realidades de la escuela, especialmente en el sector público, la conflictividad de los entornos escolares, la descomposición social que afecta a las comunidades educativas, las carencias a nivel de los recursos humanos, didácticos y locativos, el despliegue de actores 
ilegales y de bandas armadas de diverso género se constituyen en talanqueras que minimizan las posibilidades de los dispositivos legales con los que cuenta la escuela para abordar unas problemáticas sociales que desbordan sus posibilidades de atención o mitigación.

Los desarrollos jurídicos no son suficientes si no se atiende la problemática social de la cual son producto las múltiples violencias que afectan a la sociedad y la escuela. Una muy buena regulación jurídica es inocua ante la penosa situación socioeconómica y de seguridad que afronta un porcentaje alto de estudiantes del país y sus familias, especialmente en el sector público. En muchos colegios tanto públicos como privados los procesos de ajuste institucional a partir de la Ley 1620 de 2013 han servido no solamente para reformar o actualizar el manual de convivencia sino para ir más allá, repensar y reelaborar el currículo y el Proyecto Educativo Institucional. En otras instituciones no se ha pasado de un ejercicio formal que no ha tenido ningún impacto; lo grave es que estas son la mayoría.

Se requiere que los docentes conozcan y valoren críticamente los términos que la Ley 1620 de 2013 estableció para el sector y los colegios. Existe desconocimiento de la ley por parte de los docentes; por lo tanto, no se han valorado en toda su dimensión los elementos de orden conceptual, técnico, pedagógico y administrativo que introdujo la referida ley al ordenamiento educativo colombiano; esto sucede tanto en el sector público como en las instituciones educativas de carácter privado.

El parámetro desde los cuales las autoridades públicas establecen el número de docentes por institución y por número de estudiantes es restrictivo respecto a las necesidades que tienen los colegios, en el sector oficial, para atender una problemática convivencia y de violencia creciente. El Decreto 1850 de 2002, por ejemplo, fue regresivo respecto al número de docentes por número de estudiantes, comparativamente con lo que existía anteriormente ${ }^{6}$.

Cada institución educativa presenta una dinámica educativa particular, unas complejidades, un uso de los tiempos y espacios que muchas veces no son entendidas por las instituciones externas que periódicamente llegan a los colegios oficiales con la pretensión de orientar o redireccionar la política de convivencia escolar. Estas entidades, contratadas desde la administración central,

6 COLOMBIA. MINISTERIO DE EDUCACIÓN NACIONAL (2002). Decreto 1850 de Agosto 13 de 2002. por el cual se reglamenta la organización de la jornada escolar y la jornada laboral de directivos docentes y docentes de los establecimientos educativos estatales de educación formal, administrados por los departamentos, distritos y municipios certificados, y se dictan otras disposiciones. 
tienen dificultades para reconocer las especificidades institucionales y de su población, y en esa medida son poco lo que pueden hacer.

Aprietos se evidencian a diario en todas las instituciones oficiales, por ejemplo del Distrito Capital, al hacer las remisiones de estudiantes a las Empresas Prestadoras de Salud (EPS) para la atención en salud mental, son recurrentes las situaciones en que se niega la atención, se demora exageradamente la programación de una cita (4 a 6 meses) o se argumenta la falta de agenda. Igualmente el trabajo desborda la capacidad de entidades como el ICBF o las comisarías de familia; la congestión en la atención de casos dificulta la oportuna intervención o apoyo que se solicita para los estudiantes remitidos desde los departamentos de orientación escolar. De otra parte, cuando se solicita la intervención de la Policía de Infancia y Adolescencia, ante una necesidad o eventualidad, casi nunca llega al colegio, y no se tiene el pie de fuerza suficiente para la vigilancia de los entornos o zonas contiguas a las instituciones educativas dada la situación de inseguridad que padecen los estudiantes en localidades como Suba, Los Mártires, San Cristóbal o Engativá en Bogotá7.

\section{CONCLUSIONES}

La Ley 1620 de 2013 representa un hito del derecho educativo colombiano. Su aprobación por parte del Congreso de la Republica constituye una respuesta del establecimiento a los requerimientos que demandaba el sector educativo y la sociedad colombiana para atender una creciente violencia en la escuela que afecta tanto los derechos humanos, sexuales y reproductivos de millones de niños, niñas y jóvenes vinculados a la educación formal en los niveles de preescolar, básica y media, como las posibilidades de que el sistema educativo cumpla con los objetivos y metas definidos en el marco de las respectivas políticas públicas sectoriales.

El debate que ha circunscrito los ajustes institucionales exigidos por esta norma, como es natural, ha tenido que ver con dos asuntos: de una parte, sobre

7 Integraron el grupo de orientadoras escolares que colaboró en este ejercicio, las siguientes profesionales: Rosa Elena Villa Figueroa, psicóloga, especialista en intervención sistémica de la familia, con 25 años de experiencia en instituciones educativas de la Secretaría de Educación Distrital. Martha Patricia Castro Bermúdez, psicopedagoga, especialista en comunicación educativa, con 27 años de experiencia en la Secretaría de Educación Distrital. Elsa María Sierra Figueroa, trabajadora social, especialista en comunicación educativa, con 24 años de experiencia en instituciones educativas de Bogotá. María Helena, Beltrán Villegas, trabajadora social, con especialización en intervención sistémica de la familia, con 24 años de experiencia en instituciones educativas de la Secretaría de Educación Distrital. 
los alcances y posibilidades de sus disposiciones para resolver los conflictos escolares, mitigar los hechos de agresión entre pares y trasformar las prácticas pedagógicas dentro de los establecimientos educativos en la línea de asegurar mejores ambientes de trabajo escolar; de otra, sobre el origen, naturaleza y trascendencia de los factores generadores de la violencia escolar y de la violencia en la escuela.

La Ley 1620 del 2013 creó un sistema no solamente para atender los hechos o situaciones de violencia escolar sino que básicamente lo define y estructura para desarrollar acciones de promoción de los derechos humanos sexuales y reproductivos, para prevenir las situaciones de violencia en la escuela y para hacer los seguimientos que cada caso requiera de acuerdo a su complejidad o gravedad. El referido sistema integra numerosas instituciones y organismos del Estado, a nivel nacional: Ministerio de Salud, Ministerio de Cultura, Ministerio de las TIC, ICBF, Ministerio Público, Defensoría del Pueblo, Sistema de Responsabilidad Penal de Adolescentes, Policía de Infancia y Adolescencia; hace lo propio en los otros subniveles político administrativos (departamentos, distritos y municipios certificados en educación), dándole forma a los respectivos subsistemas y organismos, como los comités de convivencia escolar, hasta llegar a la unidad básica: la institución educativa.

Las políticas, estrategias y acciones de carácter convivencial estarán direccionadas por los comités de convivencia como órganos colegiados en cada nivel político administrativo (nación, departamentos, distritos y municipios) con representación de las entidades que hacen parte del sistema; en los colegios el comité escolar de convivencia, como un organismo democrático en el que tienen representación todos los estamentos de la comunidad educativa, asumirá la responsabilidad de implementar la Ruta de Atención para la Convivencia Escolar que desde sus diferentes componentes (promoción, prevención, atención y seguimiento) se constituye, junto al Sistema de Información Unificado de Convivencia Escolar, en la principal herramienta del sistema.

Se incorpora a la estructura administrativa del sector educativo colombiano un sistema cuyo eje central está en función de responder a los requerimientos en materia de convivencia de las instituciones educativas y de atención a las situaciones de acoso y de violencia que en ellas se presenten. Constituye un verdadero reto que el sistema funcione, que las autoridades públicas manifiesten voluntad y desplieguen todos los recursos necesarios para hacer del sistema un instrumento eficaz para cumplir con los propósitos que en la formalidad de los textos jurídicos y documentos de política pública se pretende alcanzar en materia de acceso, permanencia, pertinencia y calidad de la educación. 


\section{REFERENCIAS}

\section{Doctrina}

A. M. Manjarrés (2012). Violencia interpersonal, diferentes formas de presentarse: ¿es prevenible y evitable? En: Artículos complementarios, Instituto Nacional de Medicina y Ciencias Forenses. Disponible: http://www.medicinalegal.gov.co/ documents/10180/34616/9-F-11-Articulos.pdf/2e02c9b6-c22c-4f12-a9a2$9 \mathrm{e} 41 \mathrm{~b} 7 \mathrm{c} 5 \mathrm{~d} 632$

H. Bocanegra (2017). Violencias, segregación en la escuela y derecho a la educación: acercamiento conceptual y a su realidad en Colombia. En: Revista Republicana nro. 22; (enero junio); pp. 99-129.

C. Berger \& C. Lisboa (editores) (2009). Violencia escolar. Estudios y posibilidad de intervención en Latinoamérica. Santiago de Chile.

D. A. Barragán Ronderos (2016). La construcción de la mentalidad democrática como necesidad en el posconflicto. En: Revista Via Inveniendi et Iudicandi. Vol. 11, nro. 1 (enero-junio); pp.37-57.

Departamento Administrativo Nacional de Estadística, DANE (2012). Encuesta de convivencia escolar y circunstancias que la afectan, para estudiantes de $5 .^{\circ}$ a $11 .^{\circ}$ de Bogotá 2011. Bogotá, D. C. Disponible: http://www.dane.gov.co/files/investigaciones/ boletines/educacion/bol_ConvivenciaEscolar_2011.pdfde 2012.

D. Olweus (1998). Conductas de acoso y amenaza entre escolares. Madrid: Ediciones Morata.

F. Cerezo Ramírez (2008). Acoso escolar. Efectos del bullying. Boletín de la Sociedad de Pediatría de Asturias, Cantabria, Castilla y León. Bol Pediatr; 48: 353-358.

El Tiempo.com (20 de abril de 2017). Ministerio de Educación rindió cuentas. Yaneth Giha, acompañada de otros funcionarios, presentó cifras, resultados y proyectos de 2016.

Disponible: http://www.eltiempo.com/vida/educacion/rendicion-de-cuentas-delministerio-de-educacion-nacional-79810.

Instituto Colombiano de Bienestar Familiar, ICBF (2009). Tratados y convenios internacionales en materia de niñez y de familia. Coordinador editorial: Oficina de Comunicaciones y Atención al Ciudadano. Diagramación e impresión: Imprenta Nacional.

L. E. Castillo Pulido. El acoso escolar. De las causas, origen y manifestaciones a la pregunta por el sentido que le otorgan los actores. Universidad Distrital Francisco José de Caldas. Universidad de La Salle. Bogotá, Colombia. 2011. 
R. Parra Sandoval; A. González; O. P. Moritz; A. Blandón \& R. Bustamante (1998). La escuela violenta. Bogotá: Tercer Mundo Editores.

S. M. Molina Peláez (2017). ¿Qué busca el Estado con una política pública? Dinámica de las políticas públicas y los valores entre las instituciones estatales. En: Revista IUSTA nro. 46 (enero-junio); pp. 63-84.

\section{Legislación internacional}

Asamblea de la Sociedad de Naciones Unidas (24 de septiembre de 1924). Declaración de Ginebra sobre los Derechos del niño.

Asamblea General de las Naciones Unidas (1959). Declaración de los Derechos del Niño.

Asamblea General de las Naciones Unidas (diciembre 16 de 1966). Pacto Internacional de Derechos Económicos, Sociales y Culturales. Resolución 2200 A (XXI).

Asamblea General de las Naciones Unidas (20 de noviembre de 1989). Convención sobre los Derechos del Niño. Resolución 44/25.

Organización de Naciones Unidas. Comité de los Derechos del Niño (18 de abril de 2011). Observación General nro. 13 sobre el derecho del niño a no ser objeto de ninguna forma de violencia. CRC/C/GC/13.

\section{Legislación nacional}

Colombia. Congreso de la República. Ley 12 de 1991 (enero 22 de 1991). «Por medio de la cual se aprueba la Convención sobre los Derechos del Niño adoptada por la Asamblea General de las Naciones Unidas el 20 de noviembre de 1989». Diario Oficial 39640.

Colombia. Congreso de la República (marzo 3 de 2012). Gaceta del Congreso 87, Proyecto de Ley 201 de 2012, Cámara de Representantes, por el cual se crea el Sistema Nacional de Convivencia escolar y formación para el ejercicio de los Derechos Humanos, Sexuales y Reproductivos y la Prevención y Mitigación de la Violencia escolar. Exposición de motivos.

Colombia. Presidencia de Colombia (noviembre 27 de 1989). Decreto 2737 de 1989. Por el cual se expide el Código del Menor.

Colombia. Congreso de la República. (febrero 8 de 1994). Ley 115 de 1994. Por medio de la cual se expide la Ley General de Educación. Diario Oficial: 41.214. 
Colombia. Presidencia de Colombia. Ministerio de Educación Nacional (agosto 3 de 1994). Decreto 1860 de 1994. Por el cual se reglamenta parcialmente la Ley 115 de 1994 en los aspectos pedagógicos y organizativos generales.

Colombia. Congreso de la República (noviembre 8 de 2006). Ley 1098 de 2006. Por la cual se expide el Código de la Infancia y la Adolescencia. Diario Oficial nro. 46.446.

Colombia. Congreso de la República (marzo 15 de 2013). Ley 1620 de 2013. Por la cual se crea el Sistema Nacional de Convivencia Escolar y Formación para el Ejercicio de los Derechos Humanos, la Educación para la Sexualidad y la Prevención y Mitigación de la Violencia Escolar. Diario Oficial: 48.733

Colombia. Ministerio de Educación Nacional (septiembre 11 de 2013). Decreto 1965 de 2013. Por el cual se reglamenta la Ley 1620 de 2013, que crea el Sistema Nacional de Convivencia Escolar y Formación para el Ejercicio de los Derechos Humanos, la Educación para la Sexualidad y la Prevención y Mitigación de la Violencia Escolar. Diario Oficial: 48.910.

Concejo de Bogotá (febrero 23 de 2000). Acuerdo número 04 del 2000. Por el cual se crean los comités de convivencia en los establecimientos educativos oficiales y privados del Distrito Capital.

\section{Jurisprudencia}

Corte Constitucional (28 de octubre de 1992). Sentencia C-574 de 1992. Magistrado Ponente: Ciro Angarita Barón.

Corte Constitucional (9 de noviembre de 2006). Sentencia T-917 de 2006. Magistrado ponente: Manuel José Cepeda Espinosa.

Corte Constitucional (30 de noviembre de 2011). Sentencia T-905 de 2011. Magistrado ponente: Jorge Iván Palacio Palacio.

Corte Constitucional, Sala Sexta de Revisión (29 de julio de 2014). Sentencia T 562 de 2014. Magistrada ponente: Gloria Stella Ortiz Delgado.

Corte Constitucional, Sala Quinta de Revisión (3 de agosto de 2015). Sentencia T 478 de 2015. Magistrada ponente: Gloria Stella Ortiz Delgado. 